\title{
Research on the Corrosion Behavior of 14Cr12Ni3Mo2VN Stainless Steel in Different Concentrations of $\mathrm{NaCl}$ Solution
}

\author{
Quande Li ${ }^{1,2,3}$, Huimin Meng ${ }^{1,{ }^{*}}$, Randou $^{1}$,Xiufang Gong ${ }^{2,3}$, Bin Long ${ }^{2,3}$, Rong Ni $^{2,3}$, \\ Xianlong Gong ${ }^{2,3}$, Jun Dai ${ }^{2,3}$ \\ ${ }^{1}$ Institute of Advance Materials and Technology, University of Science and Technology Beijing, Beijin \\ g 100083, China \\ ${ }^{2}$ State Key Laboratory of Long-life High Temperature Materials,Deyang 618000, China; \\ ${ }^{3}$ Dongfang TurbineCo.,LTD, Deyang 618000, China; \\ *E-mail: menghm16@126.com
}

doi: $10.20964 / 2020.01 .26$

Received: 9 September 2019 / Accepted: 29 October 2019 / Published: 30 November 2019

\begin{abstract}
The corrosion behavior of $14 \mathrm{Cr} 12 \mathrm{Ni} 3 \mathrm{Mo} 2 \mathrm{VN}$ martensitic stainless steel at different chloride ion concentrations was investigated by using the open circuit potential,electrochemical impedance spectroscopy,potentiodynamic polarization,Mott-Schottkycurves,scanning electron microscopy, energy dispersive spectroscopy and laser scanning confocal microscopy. It was found that with increasing concentrations of $\mathrm{Cl}^{-}$, the open circuit potential constantly shifted in the negative direction, and the pitting potential, impedance value and protection potential of the passivation film all continuously decreased. Meanwhile, the main density $\mathrm{N}_{d}$ of a passivation film was obviously enhanced, and the number of point defects inside the passivation film and channels for corrosion medium transmission also increased.The corrosion resistance of thematerial continuously decreased with an increase in the concentration of $\mathrm{Cl}^{-}$, leading to an increase in thecorrosion tendency of the steel. $\mathrm{Fe}$ and $\mathrm{Cr}$ in a corrosion pit selectively dissolved.Fe migrated out of the pit in the form of an ion, whereas $\mathrm{Cr}$ deposited in the form of an oxide in the corrosion pit. The corrosion pattern of the material surface changed from circular pitting to linear corrosion along the material surface with increasing chloride ion concentration, but the depth of the corrosion pit did not significantly increase.
\end{abstract}

Keywords: Stainless steel; Blade;Steam turbine; Electrochemistry; Corrosion

\section{FULL TEXT}

(C) 2020 The Authors. Published by ESG (www.electrochemsci.org). This article is an open access article distributed under the terms and conditions of the Creative Commons Attribution license (http://creativecommons.org/licenses/by/4.0/). 